\title{
Comparative Reliability of Inkjet-Printed Electronics Packaging
}

\author{
T. Tilford, S. Stoyanov Senior Member IEEE, J. Braun,
}

\author{
J. C. Janhsen, M. K. Patel and C. Bailey Senior Member IEEE
}

\begin{abstract}
This paper compares the thermomechanical behavior of 3D inkjet printed microelectronics devices relative to those fabricated from traditional methods. It discusses the benefits and challenges in the adoption of additive manufacturing methods for microelectronics manufacture relative to conventional approaches. The critical issues related to the design and reliability of additively manufactured parts and systems stem from the change in the manufacturing process and the change in materials utilized. This study uses numerical modelling techniques to gain insight into these issues. This article is an extension of a paper on the same topic presented at the 2018 IEEE Electronics Packaging Technology Conference [1]

An introduction providing an overview of the area, covering salient academic research activities and discussing progress towards commercialization is presented. The state-of-the-art modular microelectronics fabrication system developed within the EU NextFactory project is introduced. This system has been used to manufacture several test samples, which were assessed both experimentally and numerically. A full series of JEDEC tests showed that the samples were reliable, successfully passing all tests.

The numerical model assessing the mechanical behavior of an inkjet-printed structure during layer-by-layer fabrication is presented. This analysis predicts that the stresses induced by the UV cure process are concentrated toward the extremities of the part and, in particular, in the lower layers which are constrained by the print platform.

Subsequently, a model of a multilayer microelectronics structure undergoing JEDEC thermal cycling is presented. The model assesses the differences in mechanical properties between a conventional FR4/copper structure and an inkjet-printed acrylic/silver structure. The model identified that the influence of the sintering process on subsequent material properties, behavior of the inject-printed structure, and reliability of the inject-printed structure is significant.

Key findings are that while stresses in the conventional and inkjet boards are relatively similar, the inkjet-printed board exhibits significantly greater deformation than the standard board. Furthermore, the mechanical stresses in the inkjet fabricated board are strongly dependent on the elastic modulus of the sintered silver material, which, in turn, is dependent on the sintering process.
\end{abstract}

Index Terms-Additive Manufacturing, Inkjet Printing, Microelectronics Fabrication, Reliability, Numerical Analysis

This article was submitted for review on the 22nd of June 2020

T. Tilford, S, Stoyanov, M. K. Patel and C. Bailey are with the School of Computing and Mathematical Sciences, University of Greenwich, London SE10 9LS, UK (email:T.Tilford@gre.ac.uk) J. Braun and J.C. Janhsen are with the Fraunhofer-Institute Manufacturing Engineering and Automation IPA, Nobelstr. 12, 70569 Stuttgart, Germany.

\section{INTRODUCTION}

A DDITIVE manufacturing is becoming increasingly widely adopted across a range of industrial sectors for the fabrication of high value and high complexity products. Piezoelectric drop-on-demand inkjet printing systems can be used to form truly three-dimensional, multi-material objects with very high dimensional accuracy. The introduction of conductive silver nano-particle inks has enabled this process to be applied to the manufacture of microelectronics systems.

Inkjet based microelectronics fabrication systems typically have multiple print heads to allow the deposition of an acrylic insulating layer patterned with silver conductive tracks. Pickand-place systems allow the integration of active and passive components within the printed structure. The flexibility of the manufacturing system can be enhanced by adopting a modular approach, integrating three-dimensional inkjet printing, pickand-place, material dispense [2], 3D inspection [3] and condition-based monitoring [4-7] processes. Additive manufacturing systems enable a wide range of microelectronics packages to be formed on a single machine in an extremely rapid process. The ability to transition from CAD design to a functional product within a few hours can be used to profoundly alter how companies approach prototyping and development of commercially saleable products.

\section{REVIEW}

A large body of academic research has been published on inkjet printing of microelectronics systems. The key enabler of the fabrication approach is the ability to accurately print and sinter conductive materials. Early academic research in this field investigated the viability of several different approaches and materials to form reliable conductive tracks. Teng et al. [8] pioneered the use of inkjet printing of microcircuits. The research used an impulse inkjet system to print a silver neodecanoate metallo-organic decomposition (MOD) ink to form a metallization grid for solar cell fabrication. The MOD inks used were particle-free solutions that were processed in a thermal furnace post-print to form the final metallic structures. A somewhat similar approach was also utilized, more recently, 
by Smith et al. [9, 10]. Teng et al. [11] subsequently extended the inkjet printing technique to rapid prototyping of electronics packages, a task which inkjet fabrication is used for today. Other researchers, e.g. Dziedzic et al. [12] advanced and extended the original technique in the field of inkjet-based solar cell fabrication.

In addition to MOD inks, several other materials were studied. Sirringhaus et al. formed high-resolution transistor circuits using PEDOT conductive polymer inks [13]. The approach adopted was based on the concept of constraining a water-based conducting polymer material to a patterned hydrophilic substrate. The substrate was prepared using photolithography and plasma etching to form the desired pattern. The conductive polymer was inkjet printed over the hydrophilic pattern and subsequently dried. This approach, therefore, augmented the accuracy of the inkjet printing process through substrate patterning.

Researchers have also studied the use of inkjet-printed conductive polymer materials to form electronics systems and/or packaging, including Burns et al. [14] who inkjet printed thin-film transistor circuits using PEDOT/PSS conductive polymers with surface patterning, van Osch et a.1 [15] who printed narrow conductive tracks without pre-patterning and Eom et al. $[16,17]$ who used inkjet dispensed PEDOT to form high-efficiency solar cells.

One of the key developments in inkjet fabrication of microelectronics systems was the adoption of nanoparticulate materials. The majority of modern inkjet printable conductive inks use sinterable nanoscale silver particulate inks. Gamota et al. [18] developed a method to print tracks at resolutions down to $100 \mu \mathrm{m}$ using a piezoelectrically driven printhead to deposit metallic nanoparticle suspensions. The ink was based on a toluene solvent with $30-50 \%$ by weight gold particles ranging 5-20 $\mathrm{nm}$ in diameter. The ink was thermally processed in an oven post-print to drive nanoparticle sintering. The work of Chung et al. [19, 20] also focused on printing tracks using dropon-demand inkjet printing techniques. In this body of work, the ink was sintered using a laser-based process. The technique was used for forming tracks on flexible substrates [21] and passive components [22].

The work of Perelaer et al. [23] focuses on inkjet printing of conductive silver tracks on a polyimide substrate. This combination of materials has become the most common in current inkjet-printed electronics applications. Perelaer used microwave irradiation as a sintering process. This is highly efficient and self-stabilizing as the unsintered silver particulates absorb electromagnetic radiation while fully sintered material reflects energy at microwave frequencies. The polymer substrates used in the fabrication process are effectively transparent to the microwave radiation, so they are not directly affected by the process. While the adoption of microwave processing techniques for the fabrication of microelectronics packaging is relatively rare, studies have demonstrated that the technique can be highly advantageous [24-27].
More recently, many researchers have been focusing on nanomaterials and nanostructures into the formulation of printable ink materials. For example, Torrisi et al. [28] and Li et al. [29] study inkjet printing of graphene-based electronics while Tortorich et al. [30] and Chen et al. [31] study inkjet printing of carbon nanotube electronics and sensor systems. Zhang et al. [32] and subsequently Cummins and Desmullliez [33] present more comprehensive reviews on the subject area.

Processes currently utilized in inkjet printing for microelectronics fabrication are typically based on multiple MEMS type inkjet printheads printing differing (insulating/conductive/support) materials at high resolution. Inks are usually based on a combination of a solvent with silver nanoparticles. The sintering process can be induced using methods including thermal (convection oven), photonic and laser sintering approaches.

One of the key challenges in inkjet printing of nanoparticulate inks is to stabilize the particles in the fluid to avoid agglomeration, which could lead to clogged nozzles [34]. This is done by stabilizing shell molecules around the nanoparticles. However, the organic stabilizers act as an insulating shell after the deposition of the ink. Thermal energy is needed to break the organic shell to enable sintering of these in a second step.

Key research into applications of piezoelectric drop-ondemand inkjet printing for additive manufacturing of microelectronics systems includes the pioneering work performed by researchers based at the Tampere University of Technology. Initial research carried out by Mäntysalo et al. [35] focused on using inkjet-printed, laser sintered, silver nanoparticulate inks alongside insulating polymer materials for system in package. Subsequent work focused on printing dielectric layers [36] electrical interconnects [37] and RF systems [38]. More recently the group have focused on inkjet printing biosensors onto flexible substrates for medical applications [39, 40].

Further relevant work includes that of Kawahara et al. [41] who utilized inkjet fabrication for rapid prototyping. The ability to use additive manufacturing processes to rapidly develop multiple prototypes of a microelectronics system or, more commonly, a PCB to evaluate a design before moving to large scale production is now being more widely adopted in the microelectronics sector. The key issue with this approach relates to how the differences in materials and material properties between AM prototyping materials and traditional FR4/copper is considered in the identification of an optimal/suitable design.

Felba et al. [42, 43] studied inkjet printing of electrical interconnects and vias on flexible substrates. The subsequent work by Felba and his research group have studied the reliability and microstructure of sintered silver interconnects which strongly influence the reliability of the overall microelectronics system. Faller et al. [44-46] focused on the use of inkjet-printed sintered silver inks to form force/pressure sensors and MEMS devices. 
Arias et al [47] study the use of flexible printed electronics for medical monitoring, presenting a detailed fabrication process for systems combining sensors, data processing and wireless transmission. The approach is intended to support development of flexible medical devices able to monitor vital signs, such as body temperature, heart rate, respiration rate and blood pressure for applications such as both fitness monitoring and medical diagnostics. Arias, Poliks et al [48] extend upon this work to consider the challenges in interfacing the hard and soft electronics components. Flexible, 'soft', sensors are required to interface with the human skin while 'hard' semiconductor devices are required for tasks such as data processing. A single-substrate interfacing approach is presented which provides an alternative to the more traditional multisubstrate approach with its associated interconnect reliability issues.

The reliability theme is continued by the work of Hackler et al [49] supported by the US FlexTech consortium [50] who discuss the need for reliability qualification standards for printed electronics that differ from the traditional JECEC and MIL standards used for conventional electronics reliability assessment. Sitaraman et al [51] assess the mechanical and electrical behaviour of the printed silver conductor under the adaptive curvature flexure test. This work has a strong correlation with the findings of this paper as we, unsurprisingly see decreases in electrical conductivity of the printed silver tracks as thermal cycling progresses. The work in this paper indicated that the magnitude of deformation of additively manufactured systems is greater than that of conventionally manufactured systems. While the degree of curvature the test samples in this work were subjected too is far below that assessed in the Sitaraman study, a reduction in electrical conductivity as the system ages would be expected and should be quantified.

The effect of sintering profile on long term reliability of printed silver tracks is becoming an area of interest, with e.g. Lall et al [52] looking at the influence of sinter temperature on fatigue life and Roshanghias et al [53] studying how differing sintering strategies influence conductor microstructure.

These academic studies have now been augmented by several commercially available systems intended for production of saleable products. Key examples of commercial fabrication systems based on inkjet dispense of multiple materials to enable fabrication of complex microelectronics products are the Nano Dimension Dragonfly [54] and the Optomec [55] systems.

The EU funded NextFactory [56] project has developed a modular hybrid machine setup for the manufacture of microelectronics systems integrating three-dimensional inkjet printing, micro-deposition, micro-assembly, and a curing system in four process modules.

Module 1: 3D-Inkjet-printing is used for effective material deposition. Using different piezo printheads (4x Fujifilm Dimatix SL-128 and 8x Fujifilm Dimatix QS-256), versatile material arrangements - especially conductive and nonconductive materials - are possible. A UV lamp (Phoseon FE400, $\lambda=395 \mathrm{~nm}, \mathrm{I}=8 \mathrm{~W} / \mathrm{cm}^{2}$ ) allows prepinning of printed photo polymeric inks. The precise adjustment of the printed layer height is achieved by a roller based flatting unit.

Module 2: Different curing processes like wideband UVcuring (OmniCure S2000, P = 200W) and temperature-based (IR, NIR - Adphos custom module with 9 emitters, $\mathrm{P}=19 \mathrm{~kW}$ ) curing methods are integral part of the NextFactory process chain, in order to process different types of materials - e.g. particle based silver inks in direct combination with acrylic insulating materials.

Module 3: Flexible micro-assembly processes allow for component placement directly onto - respectively into - the printed 3D-substrate. This gives the potential to boost the functionality of the printed product, as the functional scope of the overall part is no more limited to printable functionality. Instead, pre $\neg$ manufactured parts like electronic components, sensors or micro-actuators can become an integral part of the overall system. To further broaden the range of applicable materials, robust and versatile dispensing technologies are used to complement the effective inkjet-technology for high-viscous, non-printable materials. Thus, state-of-the art materials like conducting glues or fillers can be integrated.

Module 4: Inline inspection methods based on camera systems are used for process monitoring, quality prediction and process alignment. As the overall hybrid process chain achieves an increased degree of complexity (e.g., in comparison to stateof-the-art AM technologies), this way of continuous monitoring is inevitable to set up a stable process without the need for timeconsuming, product-dependent optimization processes.

The machine can accurately deposit and cure both functional and structural inks and place/embed components in an integrated manner within a single platform. This system, illustrated in Figures 1 and 2, uses a hybrid approach to increase its flexibility, with an inkjet system augmented by microdeposition tools that enable conductive adhesive materials to be used alongside silver nano-inks for conductive features.

The system enables producers of micro-mechatronic systems to manufacture complete products on a single machine with the manufacturing process from CAD design to the finished product taking several hours rather than more lengthy timescales typically associated with traditional manufacturing methods. The three-dimensional nature of the build process enables the manufacture of complex 3D microsystems as readily as $2 \mathrm{D}$ and $2.5 \mathrm{D}$ devices.

At the moment the NextFactory concept has a TRL level of 7-8 and is therefore predominantly used as a research and technology platform to further investigate the inline hybrid process chain e.g between functional 3D inkjet printing and assembly for micro-mechatronic systems. This allows the highest degree of freedom for process development as well of interacting processes e.g. the effects of various curing strategies to avoid degassing of printed materials as well as the influence of the different sintering methods (IR, NIR) to discrete mounted components like ICs and printed substrates. 


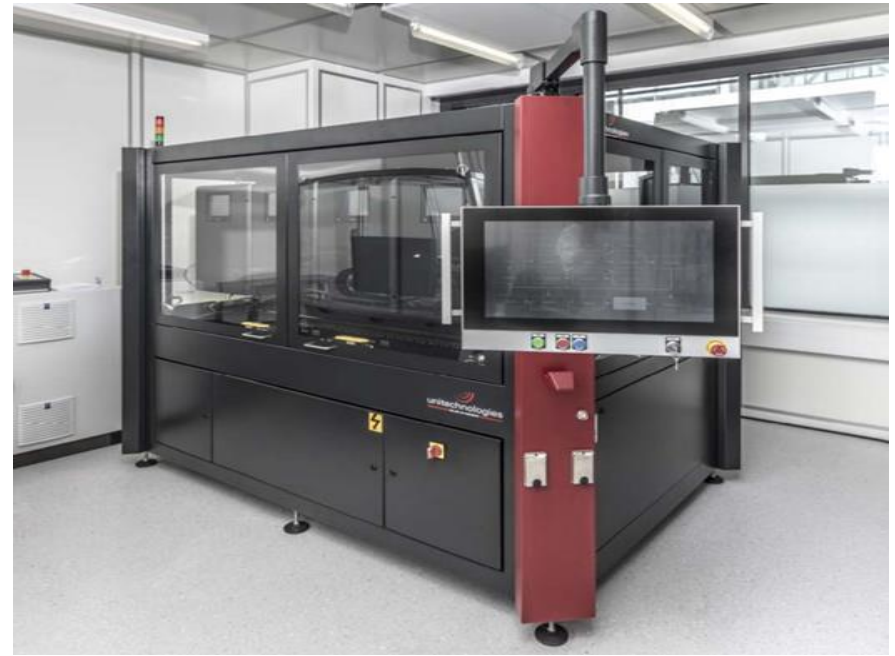

Fig. 1. NextFactory modular 3D manufacturing system

With the transition from lab-based research systems to the manufacture of commercial products comes the issues of ensuring reliability and robustness of the fabricated parts. The electronics sector is rightly conservative in its approach to ensuring the reliability of commercial products, with tests regimes such as JEDEC [57] utilized to effectively evaluate components and systems.

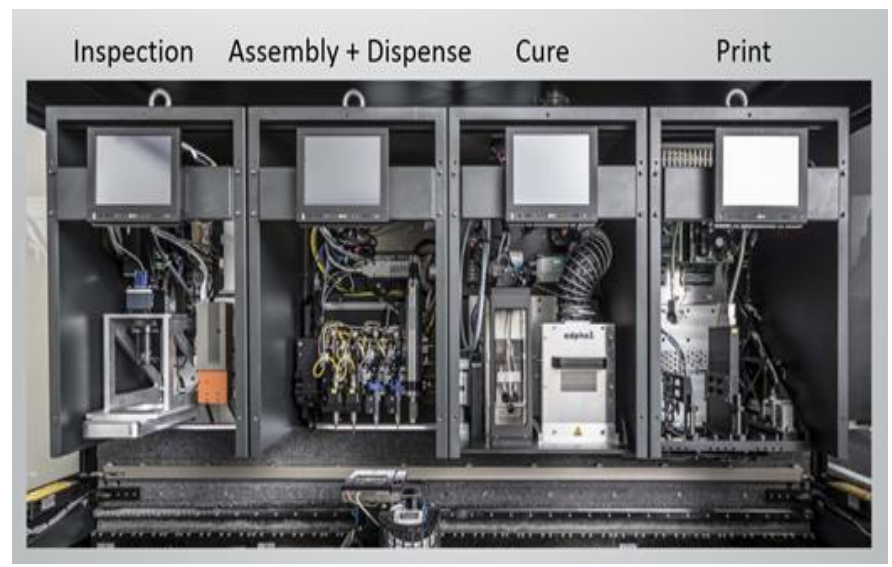

Fig. 2. Interior view of the NextFactory 3D manufacturing system showing modular nature of printing, cure, assembly, dispense and inspection

The reliability of test structures, illustrated in Figure 3, manufactured using the NextFactory system, has been assessed through comprehensive JEDEC testing. The results of these tests, described in [58], indicate that the products were reliable with all tests passed.

\section{A. Indicative manufacturing process}

While the inkjet manufacturing systems mentioned in previous sections have, by no means, a standardized assembly process, there are typically several common elements. A description of a generalized indicative inkjet fabrication process can be broken into a number of steps;

The first step, depicted in Figure 1(a), is to deposit several layers of support material onto the printing baseplate. This material is soluble, typically in either acetone, as used in the
NextFactory approach, or a Sodium Hydroxide solution. Printing the first layers from support material ensures the printed part can be readily detached from the baseplate when required.

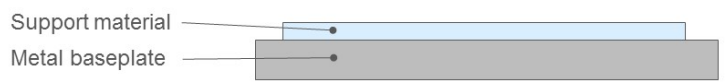

Fig. 1(a). Deposition of a support material layer onto the aluminium baseplate.

Subsequently, layers of insulating material are deposited as depicted in figure 1(b). The pattern for each layer and the printing route taken by the print head is defined in Bitmap file. The acrylic material is pinned by a low power LED lamp $\lambda=395 \mathrm{~nm}, I=4 \mathrm{~mW} / \mathrm{cm}^{2}$, the layer height is levelled by a roller based fattening unit ( $h=10-20 \mathrm{um}$ ) and finally cured using a mercury UV lamp, typically, at each layer. Alternatives involving partial cure of layers and cure after $n$ layers are also possible.

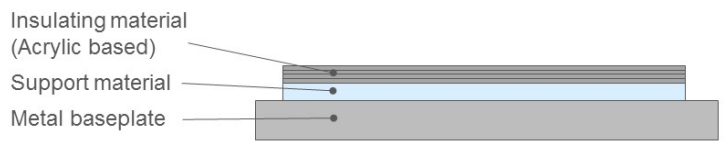

Fig. 1(b). Deposition of an acrylic insulating material layers onto the initial support material layer.

Microelectronic packages are often integrated into the structure in an embedded manner (figure 1(c)). The print strategy can form cavities for the embedded components where required.

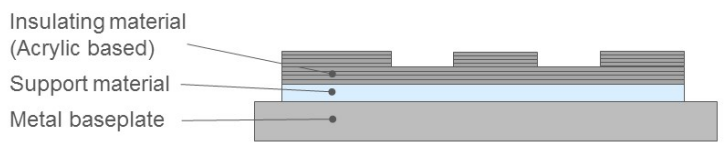

Fig. 1(c). Formation of cavities for embedded components within printed insulating material layers.

Conducting tracks are formed from silver nano-particulate inks (figure 1(d)). These are inkjet printed and can be sintered using methods such as IR, NIR, photonic or laser. The NextFactory system uses the Near Infra-Red approach. Where components are embedded, the tracks are printed up to the edge of the cavity and terminate in a pattern corresponding to the package interconnects.

One of the key messages that this article attempts to convey, is that the quality of the sintering process defines the thermomechanical properties of the silver conductive tracks which substantially influence the thermomechanical behaviour and subsequently the reliability of additively manufactured electronics systems. The optimal sinter profile is clearly related to the materials utilised. There may additionally be a multiobjective nature to optimisation of the profile with trade-offs in e.g. resistance vs mechanical factors. 
As a guide, three sintering profiles were found to work reasonably well with the materials used; two passes with four $5,4 \mathrm{~kW}$ lamps scanning at $10 \mathrm{~m} / \mathrm{min}$, one pass with four $5,4 \mathrm{~kW}$ lamps scanning at $6 \mathrm{~m} / \mathrm{min}$, two passes with four $5,4 \mathrm{~kW}$ lamps scanning at $15 \mathrm{~m} / \mathrm{min}$. However, the sinter profile will clearly need to be optimised based on material selection and system setup.

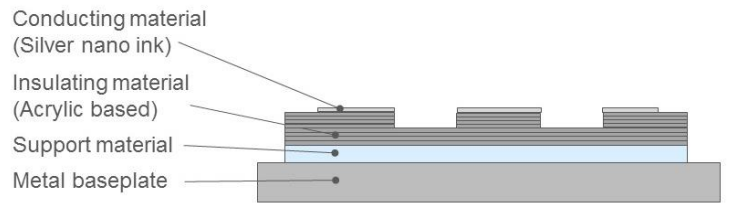

Fig. 1(d). Printing and sintering of silver ink to form a pattern of conductive tracks on the surface of the insulating material

After the sintering process is complete, a pick-and-place system adds the required components to the structure (figure 1(e)). These can be surface mounted or embedded. These are placed with the interconnects / pads on the upper surface.

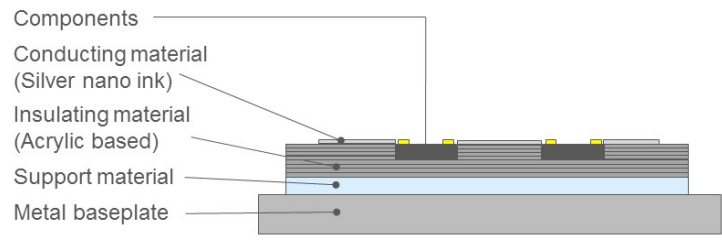

Fig. 1(e). Placement of components within the structure

The tracks are joined to the package interconnects using an isotropic conductive adhesive material (figure 1(f)). The sintered silver material is highly brittle so adoption of a more malleable material to form a bridge between the tracks and the interconnects is beneficial to the long-term reliability and robustness of the fabricated system.

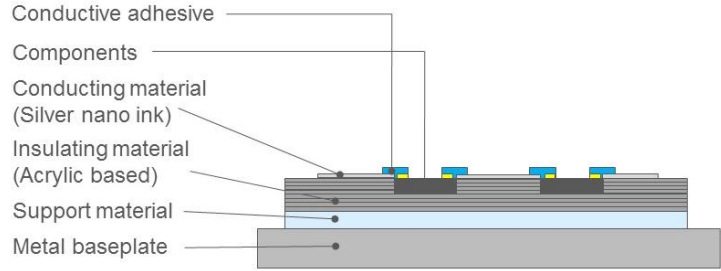

Fig. 1(f). Bridge formed from isotropic conductive adhesive between silver tracks and package interconnects.

Following Isotropic Conductive Adhesive (ICA) cure, further layers of insulating layers and conductive tracks are printed, forming a fully three-dimensional microelectronics system (figure $1(\mathrm{~g})$ ). One of the key challenges in the manufacture of microelectronics systems using this process is the formation of high aspect ratio vias.

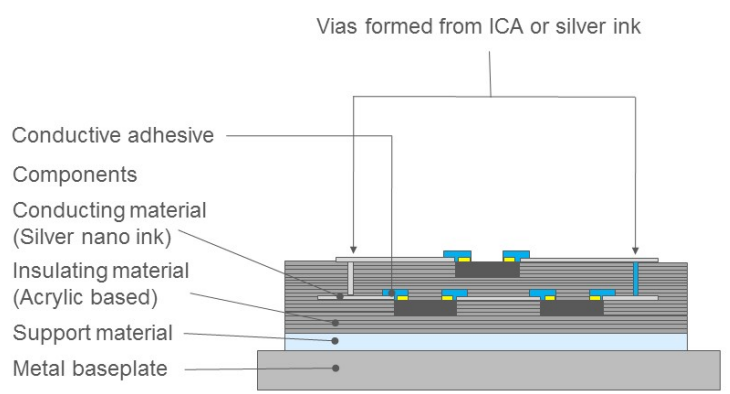

Fig. 1(g). Three-dimensional microelectronics system formed through printing subsequent layers and embedding required packages. Vias formed to connect layers.

The fabrication of the system is completed by dissolving the support materials used and separating the part from the baseplate (figure 1(h)). The structure is typically printed in an inverted state, with the final inverted part with support materials removed depicted in figure 1(i).

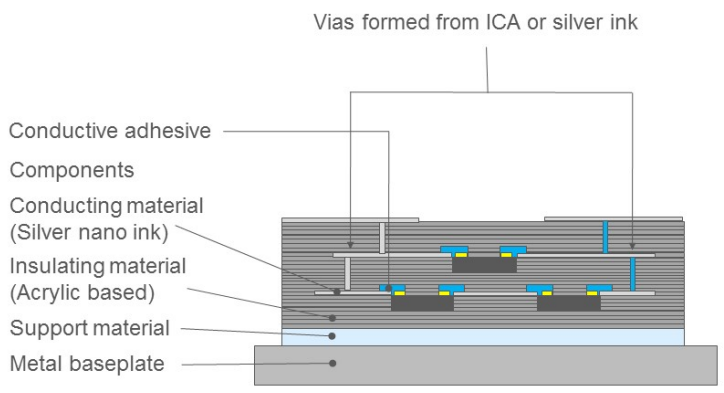

Fig. 1(h). Printing of system is finalized

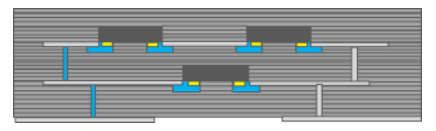

Fig. 1(i). Support materials dissolved to separate part from the baseplate. Part is inverted

\section{B. Key manufacturing Challenges}

Several key manufacturing challenges need to be addressed in order to progress the capability of inkjet fabrication of microelectronics systems. There is a continuing drive to reduce printed tack and gap dimensions through an increase in print resolution. This, however, drives down the nanoparticulate size that can be dispensed. As the particulate sintering temperature is physically correlated to the particle diameter, this brings about questions relating to how to ensure the inks do not agglomerate/sinter within the print system or printhead.

There is also a clear desire to be able to print very high aspect ratio electrical/thermal vias, which inkjet systems often struggle with. Then there is the question of sintering the nanoparticulate inks. The multiple sintering options - thermal, photonic, laser and microwave - all have different advantages and drawbacks. The sintering process for each option needs to be optimized to develop a robust and reliable conductive track.

One of the approaches to ensure overall reliability of a manufacturing process is to use AI / Machine Intelligence or condition-based monitoring to attempt to provide an 
overarching control mechanism that interprets the correlations between process parameters and several output metrics and is potentially capable of adapting the fabrication process to maximize quality (or any other metric) while the part is being built. The NextFactory system has a condition-based monitoring module $[4-7,58,59]$ which is capable of altering the build process to maximize the quality of the final product. Research on AI for improving quality of manufactured products will be of profound importance to the microelectronics fabrication sector, and indeed more widely, but a detailed discussion of the topic is beyond the scope of this article.

\section{DESIGN CHALLENGES FOR INKJET-PRINTED ELECTRONICS}

The change in the manufacturing process, from the traditional surface mount PCB to embedded inkjet printed, brings about several new design challenges. The manufacturing process is novel, and the materials involved differ significantly from traditional FR4 and copper. As such, it is necessary to redefine our understanding of optimal system design and develop new approaches to reliability analysis for products fabricated through this approach.

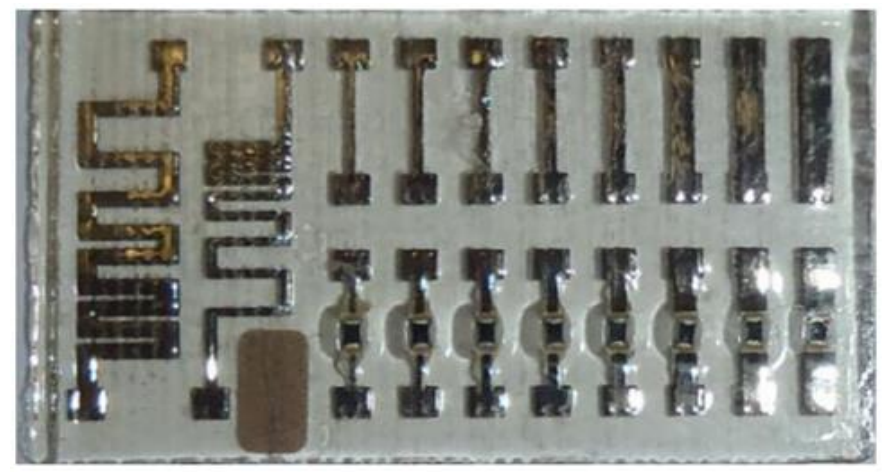

Fig. 3. Inkjet-printed test structure for JEDEC reliability evaluation

When assessing the reliability of a microelectronics structure, key areas of interest include internal stresses which result from the manufacturing process and stresses that arise from thermal and/or mechanical deformation of the structure. The layer-by-layer additive manufacturing paradigm typically involves curing each layer before proceeding to print the subsequent layer (although there are printing procedures that do not abide by this e.g. only partially curing layers or curing after several layers have been deposited). The UV curable polymer materials used in the process shrink during curing, which is a source of strain. This results in mechanical stresses that are locked into the structure as it is formed. This can influence the reliability of the structure and can/will result in post-fabrication warpage.

Additionally, the mechanical properties of the materials used differ significantly from the conventional FR4/copper that the sector is familiar with. The acrylic material is isotropic and relatively soft compared to FR4, while the properties of the conductive silver ink material tend to depend quite strongly upon the post-deposition sintering process. These issues are investigated through numerical modelling techniques to improve our understanding of these effects.

\section{NUMERICAL ANALYSIS OF PRINTED ELECTRONICS MANUFACTURING}

The layer-by-layer printing process has been modelled to gain insight into the magnitude and distribution of mechanical stresses which are generated within the part as it is fabricated. This model can subsequently be utilized to investigate the sensitivity of these stresses to several process/operating parameters. The key strain driver in this process is the shrinkage of the UV curable acrylic materials resulting from polymeric crosslinking. A Finite Element Analysis (FEA) based model has been developed, which can consider stresses with UV cured acrylic material in a layer-by-layer printing process. A more detailed outline of this model investigation can be found in [6]. This section provides a summary and overview of the main findings from that study.

The result presented here considers 10 layers of insulating material, with the thickness of single layer deposited as liquid ink defined to be 25 microns. The effects of volumetric cure shrinkage equal to $3.0 \%$ for the insulating ink following UV cure are assessed in this investigation. This simulation also assumes that UV cure takes place after each layer is deposited. As a result, the deposition of each layer of the ink material occurs on top of already cured previous layers. The analysis is isothermal and therefore only considers volumetric variation stemming from the cure process with no thermal component.

A key model assumption made here is that the first layer is deposited on a rigid substrate. Under this printing scenario, the bottom surface of the very first layer is restricted to displace inplane. Note that under the assumption for printing the first layer on a supporting material structure, often of the same material, that is subsequently removed, will require a different representation of the model boundary conditions. It is also assumed that a new liquid layer is always printed as per the target in-plane layer dimension and thickness.

The displacement results are due to shrinkage and capture all inter-layer interactions; thus, they predict the final shape and its deviation from the CAD-defined original target. Figure 4 illustrates the modelling results related to the state of the structure after printing each of the 10 layers. The contours refer to the stress and detail the stress pattern at the edge of the printed structure. The shape of the layers is the deformed shape illustrated with a magnification factor of 5 . The red dotted lines define the original target topology of the 10 layers. Finally, the grey layer is a schematic representation where 25 microns of liquid ink is deposited.

Under the model assumptions, the following main conclusions from this analysis are derived. The highest stress develops at the edge region of the first layer (due to the assumption for perfect adhesion and pinning the area of the deposited liquid ink) but away from the edge region stress is very low and can be neglected. In terms of the cross-sectional profile at the peripheral sides of the printed layers domain, the modelling results show the occurrence of inward in-plane deformation due to cure shrinkage. This in-plane shrinkage is limited as cured layers beneath provide significant restriction for deformations in this direction. An important observation from this analysis is that shrinkage affects the thickness (buildup direction) of the printed structure predominantly. 


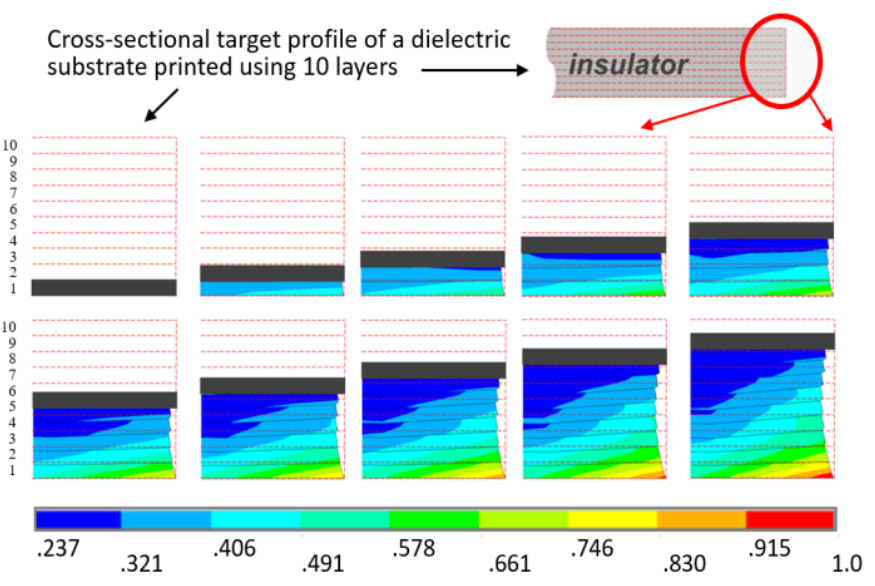

Fig. 4. Modelling predictions from finite element analysis contour plots of the normalised stress intensity (normalised over 0-1 range, corresponding to actual min-max stress range) in the 10-layer printed structure. Deformed shape of the printed structure is magnified by a factor $\mathrm{F}=5$.
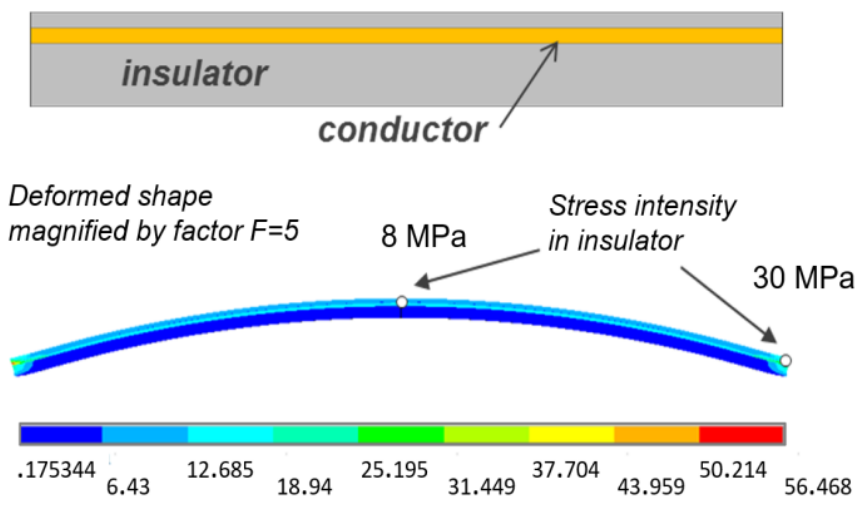

Fig. 5. Simulation results for the predicted stress intensity (MPa) distribution in a fully cured ten-layer printed structure with an imbedded conductive layer (printed layers \#7 \& \#8 counted from bottom) within the insulator. Deformed shape is magnified by a factor $\mathrm{F}=5$.

Figure 5 details another model derived result where similar 10layer printed structure, with same layer topology and thickness as the insulating structure, but this time with a 50-micron thick layer of conductive material deposited with printed layers \#7 and \#8. In this case, residual stress and deformation are affected by the difference in the cure shrinkage percentage between the insulating and conducting materials. In this model, the printed structure is assumed to be released from the substrate on top of which the inks have been printed. As a result, some of the stress built in the cured layers due to the fixture to the substrate will be relaxed. Stresses, however, are higher as a result of the stiffer conductive layer, and this introduces the risk of delamination failure. For the analysed design, the model predicted that the insulating material layers printed on top of the conductive layer are in the state of higher stress. The reliability concern in this instance would be if interfacial stress is above a critical level, i.e. exceeds the adhesion strength of the insulator, as this can result in a delamination failure at the conductive material interface.

\section{NUMERICAL ANALYSIS OF RELIABILITY TESTING}

In addition to the experimental assessment of the reliability of the inkjet-printed parts, a numerical model of a more complex multilayer design has been developed for the assessment of its behavior during thermal cycling. This consisting of a lower insulator layer, a series of conductive tracks, a second insulating layer and an upper series of tracks, A QFN package and a set of zero-Ohm resistors are mounted on the board along with a connector assembly. A CAD model of the geometry is presented in Figures 6 and 7. A numerical model of this structure was developed to assess the influence of different manufacturing approaches on the structural behavior of the sample while undergoing thermal cycling, with an inkjetprinted test structure numerically compared to a conventionally manufactured equivalent. The two manufacturing approaches are represented solely through materials used, with the 'conventional' manufacture using FR4 as the insulating material and copper as the conductor. The 'inkjet' manufacture using acrylic as the insulator and silver nano-ink as the conductor.

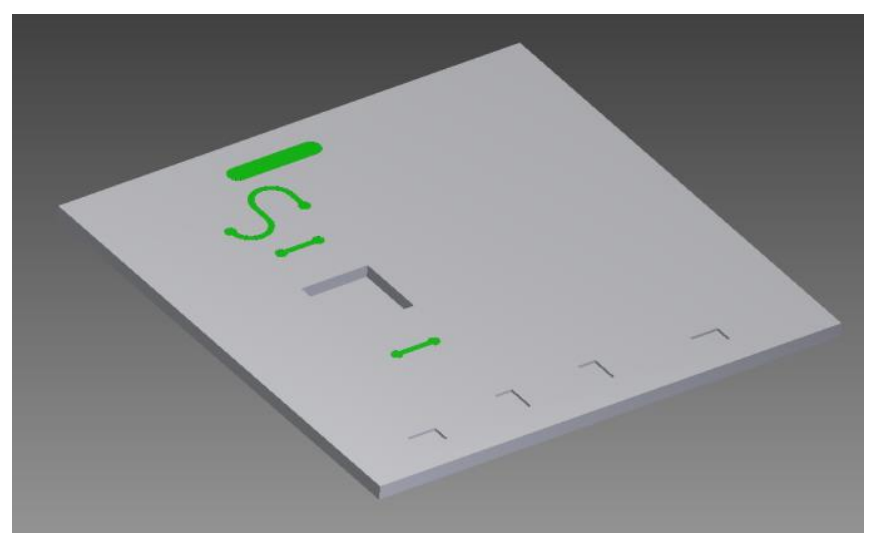

Fig. 6. Lower layer of the test structure

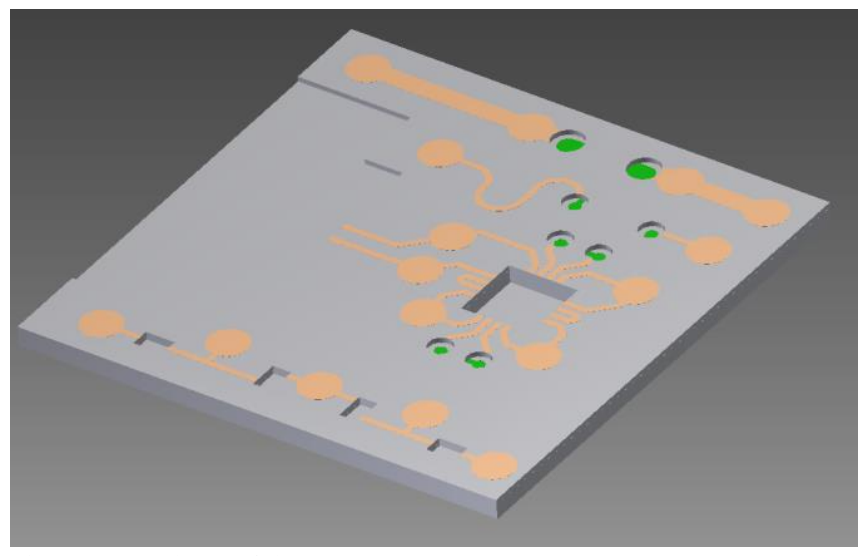

Fig. 7. Upper layer of the test structure 
The QFN, resistor and connector have been omitted from this model as these only influence the board behavior locally. Additionally, the connector is not considered as it has little to no impact on the overall mechanical performance of the structure.

The JEDEC [57] thermal cycling test is widely used as a qualification standard in the microelectronics industry. The test consists of placing the component in an oven, which varies the temperature by following- a defined temperature profile. The test profile adopted in this study, illustrated in Figure 8, comprises of two 15 minute dwell periods at $+125^{\circ} \mathrm{C}$ and -55 ${ }^{\circ} \mathrm{C}$ separated by linear 15 -minute temperature ramps.

The FR4 material is orthotropic - material properties differ between in-plane and through-plane directions, and therefore this material behavior was included in the model. All other materials are considered isotropic - properties are independent of direction. The numerical model has only considered the elastic deformation of the materials involved. The material property values used are detailed in Table 1. It should be made clear that the thermomechanical properties of the 'inkjet' materials have not been directly assessed, with values obtained from published literature. The resulting uncertainty in property values correlates to uncertainties in model predictions. This is especially relevant for the properties of the sintered silver material and will be discussed further in the following section. The material properties used for the acrylic material relate to a fully cured state. Although incomplete cure may alter these properties, this has not been considered in this work.

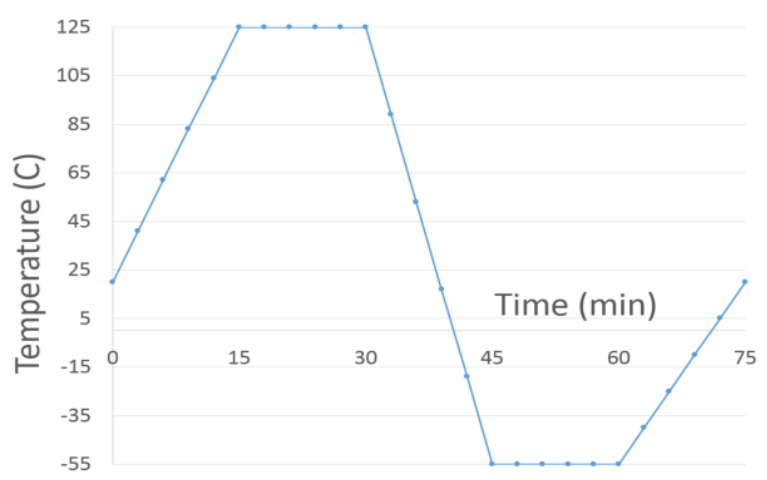

Fig. 8. Thermal cycling temperature profile

The numerical model uses approximately 4.2 million elements to describe the geometry, with relevant structural constraints imposed. A relatively fine mesh has been utilised to capture the physics at the conductor-insulator boundary, with element lengthscales at this interface in the range of 10-15 $\mu \mathrm{m}$.

Model geometry and discretization are illustrated in Figures 9 and 10. Contour plots of the total deformation and effective von Mises stresses for the 'Standard' and 'Inkjet' manufacturing processes are shown in Figures 11 and 12. Note that the total deformation is magnified by a factor of ten during the plotting process to aid clarity. Key results from the analyses are presented in Table 2.
TABLE I

MATERIAL PROPERTIES

\begin{tabular}{|c|c|c|c|c|}
\hline \multirow{2}{*}{ Parameter } & \multicolumn{2}{|c|}{ Standard } & \multicolumn{2}{|c|}{ Inkjet } \\
\hline & $\mathrm{R} 4$ & Copper & Acrylic & Silver \\
\hline $\begin{array}{l}\text { Young's } \\
\text { Modulus }\end{array}$ & $11.8 \mid 7.5$ & 128 & 3.0 & 10.0 \\
\hline $\begin{array}{l}\text { Poisson's } \\
\text { Ratio }\end{array}$ & $0.27 \mid 0.22$ & 0.34 & 0.375 & 0.37 \\
\hline $\begin{array}{l}\text { Coefficient of } \\
\text { Thermal } \\
\text { Expansion }\end{array}$ & $11 \mid 16$ & 16.5 & 73.5 & 19 \\
\hline \multicolumn{5}{|c|}{$\begin{array}{c}\text { TABLE II } \\
\text { VARIATION OF PEAK VON MISES STRESS WITH SINTERED } \\
\text { SILVER ELASTIC MODULUS }\end{array}$} \\
\hline Parameter & \multicolumn{2}{|c|}{ Standard Materials } & \multicolumn{2}{|c|}{ Inkjet Materials } \\
\hline Peak deformation & \multicolumn{2}{|c|}{$49 \mu \mathrm{M} @ 125^{\circ} \mathrm{C}$} & \multicolumn{2}{|c|}{$326 \mu \mathrm{M} @ 125^{\circ} \mathrm{C}$} \\
\hline Peak von Mises Stress & \multicolumn{2}{|c|}{$285 \mathrm{MPa}$ @ $125^{\circ} \mathrm{C}$} & \multicolumn{2}{|c|}{$256 \mathrm{MPa}$ @ $125^{\circ} \mathrm{C}$} \\
\hline
\end{tabular}

The primary findings of the study are that the peak value of the von Mises effective stress is relatively similar for the two manufacturing processes. However, the peak deformation of the 'Inkjet board is significantly greater than that of the 'Standard' board. This is considered to be due to the inkjet manufacturing approach utilizing a significantly less rigid insulator material, a significantly less rigid conductor material while combined with a substantially greater CTE mismatch when compared to the standard manufacturing approach.

A more considered approach to assessment of the physics of failure of the printed assembly would be beneficial to assess more effectively the relative reliability performance of the standard and inkjet fabrication approaches. This will require the gathering of accurate material characterisation data, further insights into the failure modes and mechanisms and development of representative non-linear material models for the assembly materials of interest, for example for the plastic behaviour of sintered silver and the viscoelastic behaviour of the insulating materials. Consequently, it will become feasible to adopt in the modelling analysis damage metrics and indicators that can help assessing the degradation of materials and the risk of failure occurrence/ time-to-failure. For example, the fatigue damage in sintered silver can be evaluated by using the volume weighted average of accumulated inelastic strain energy density or plastic strain under temperature cycling loads, and the value can be deployed as indicator of damage and also correlated to experimental life-time data. The latter will result in the development of a lifetime model for inkjet printed electronics systems, and while this is beyond the scope of the current work and article, it is something that should clearly be considered as a key area for further research. 


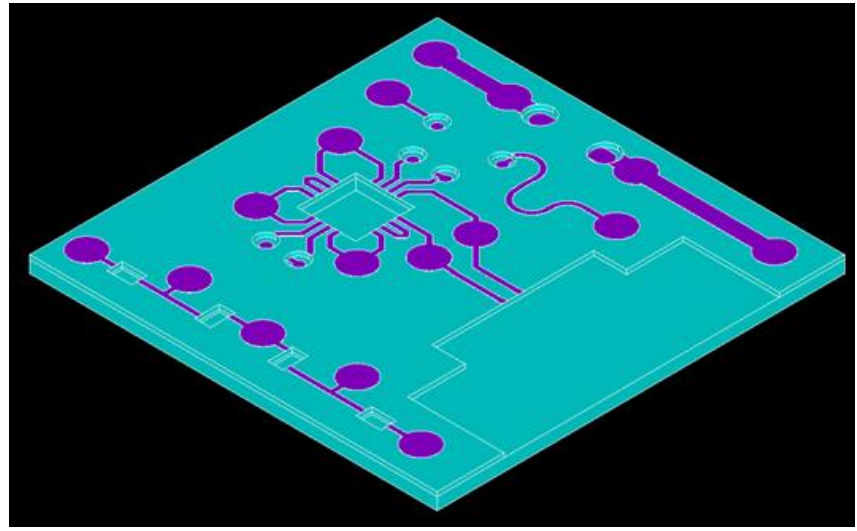

Fig. 9. Illustration of Finite Element model geometry

A critical factor that must be borne in mind when attempting to assess the thermomechanical behavior of an additively manufactured microelectronics system is the influence of sinter quality. Wereszczak et al. [60] presented data correlating the Elastic Modulus of sintered silver relative to its density. The data suggests a very significant, nonlinear, relationship between the two. The experimental data and the associated regressed function have been reconstructed and presented in Figure 13.

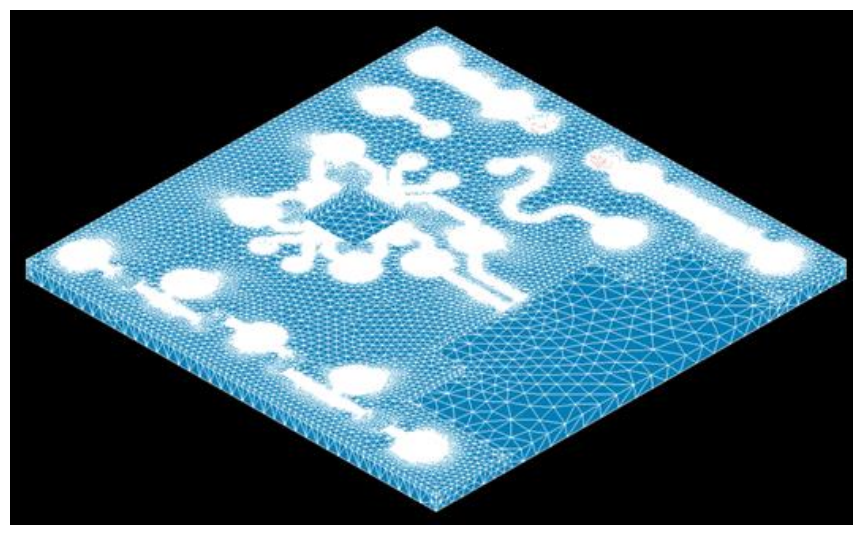

Fig. 10. Illustration of Finite Element model discretization

As the silver density is related to the quality of the sintering process, the numerical model has been used to evaluate the influence of sinter quality on the thermomechanical behavior of the additively manufactured multilayer test structure. The study comprised of running the analysis with differing silver Elastic Modulus values in the range suggested by Wereszczak et al. but with all other aspects of the analysis invariant. The peak von Mises stresses calculated at each of the four elastic modulus values assessed in the study are presented in Table III. The variation in peak stress with silver elastic modulus and hence sinter quality is significant. The quality of the sintering process should, therefore, be considered as a key factor in assessing the thermomechanical performance of additively manufactured systems and more broadly in the context of the overall reliability of additively manufactured systems.

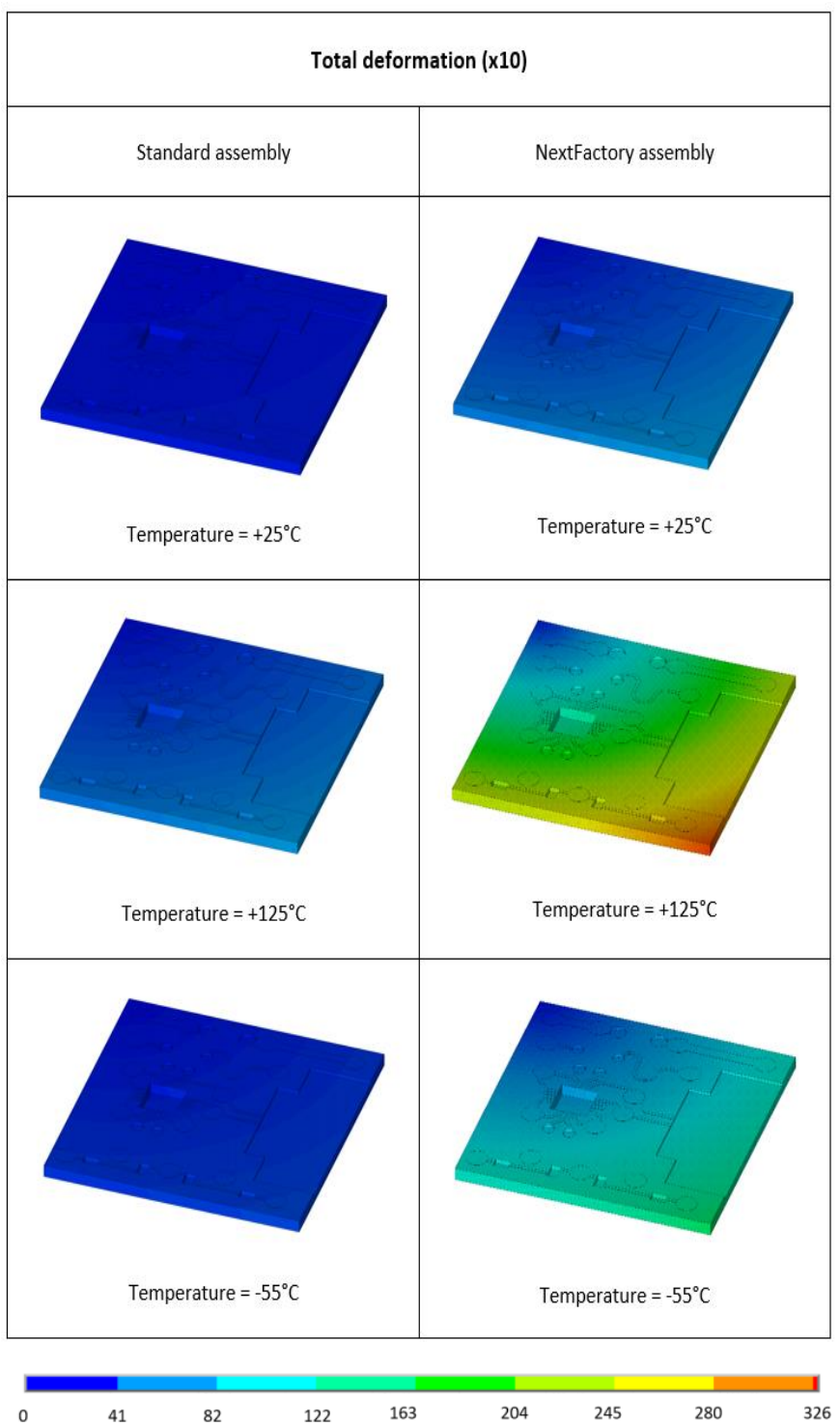

Fig. 11. Contour plots of total deformation of test structure undergoing thermal cycling, with axes units in micron. 


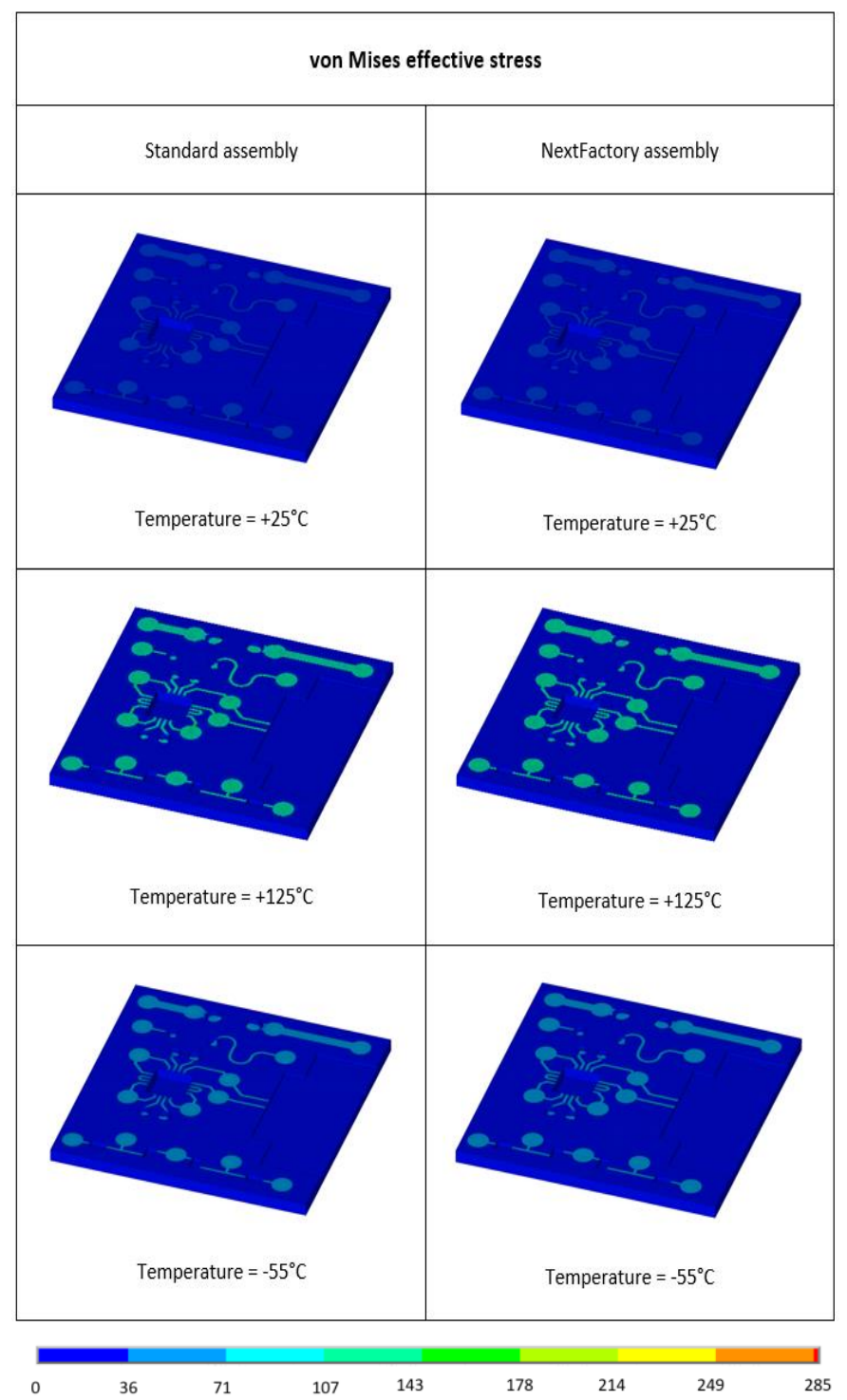

Fig. 12. Contour plots of von Mises stress within test structure undergoing thermal cycling, with axes units of $\mathrm{MPa}$.

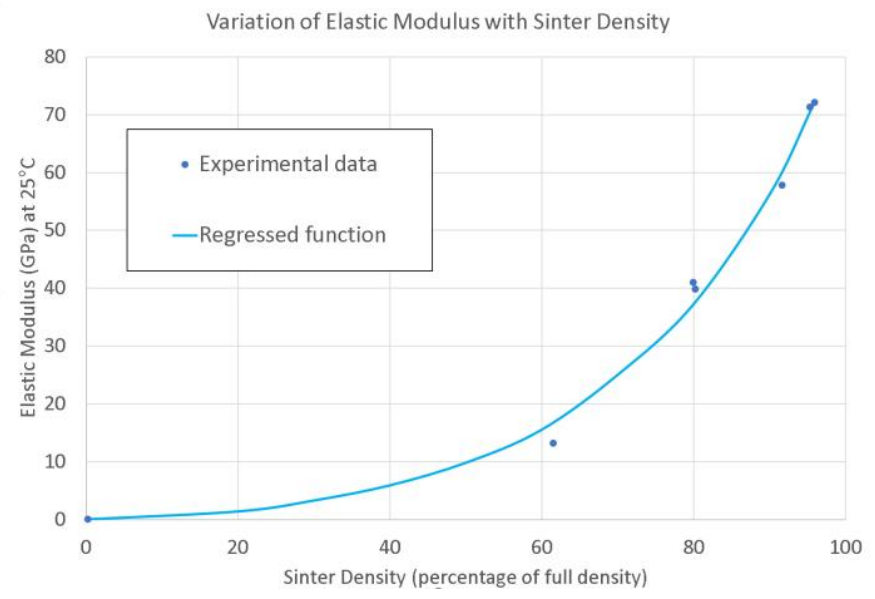

Fig. 13. Reconstructed contour plot illustrating Wereszczak ORNL data correlating variation in Elastic modulus with sinter density.
TABLE III

VARIATION OF PEAK STRESS WITH SILVER ELASTIC MODULUS

\begin{tabular}{ll}
\hline \hline Elastic Modulus (GPa.) & $\begin{array}{l}\text { Peak von Mises stress } \\
\left(\mathrm{MPa} . @ 125^{\circ} \mathrm{C}\right)\end{array}$ \\
\hline 10 & 256 \\
20 & 709 \\
30 & 1130 \\
40 & 1470 \\
\hline \hline
\end{tabular}

\section{CONCLUSIONS}

This study has utilized numerical modelling techniques to gain insight into the reliability implications of inkjet-based additive manufacturing of microelectronics systems. Insight into the stresses developed during (1) the manufacturing process and (2) subsequent thermal-cycling has been gained through the development of a model of the layer-by-layer inkjet fabrication process. Conclusions are:

- Stresses induced by the UV cure process are concentrated toward the extremities of the part and, in particular, in the lower layers which are constrained by the print platform.

- For subsequent thermal cycling, the stresses in the conventional and inkjet test structures are relatively similar, although the inkjet-printed board exhibits significantly greater deformation than the standard board.

- This study indicates that the mechanical stresses in the inkjet fabricated board are strongly dependent on the sintering process and its impact on density and Modulus.

Future research is required on the relationship between sintering processes and material properties such as density, modulus, and CTE to ensure that design and analysis tools have robust data sets for subsequent reliability predictions.

\section{ACKNOWLEDGMENTS}

The authors acknowledge the consortium in the EU-funded project NextFactory (http://www.nextfactory-project.eu/) and the European Community's 7th Framework (FP7/2007-2013) for funding this work under grant agreement No. 608985.

\section{REFERENCES}

1. Tilford, T., Stoyanov, S., Birch, R. and Bailey, C., 2018, December. Numerical Analysis of the Design and Manufacture of Inkjet Printed Electronics Packaging. In 2018 IEEE 20th Electronics Packaging Technology Conference (EPTC) (pp. 167-172). IEEE.

2. Guenthel, J., Holzinger, D., Birch, R. and Refle, O., Process \& Machine Development for the Integration of Conductive Paths Inside a 3D Printed Microsystem, Proc. Direct Digital Manufacturing Conference, Berlin, Germany, 2016

3. http://www.heliotis.ch [Accessed: 3 January 2020] 
4. Stoyanov, S. and Bailey, C., Machine learning for additive manufacturing of electronics, Proc. 40th International Spring Seminar on Electronics Technology (ISSE), 2017

5. Tourloukis, G., Stoyanov, S., Tilford, T. and Bailey, C., Predictive modelling for 3D inkjet printing processes, Proc. 39th International Spring Seminar on Electronics Technology (ISSE), 2016, pp. 257-262

6. Stoyanov, S., Tourloukis, G., Tilford, T. and Bailey, C., 2016, April. Modelling methodologies for assessment of 3D inkjet-printed electronics. In 2016 17th International Conference on Thermal, Mechanical and Multi-Physics Simulation and Experiments in Microelectronics and Microsystems (EuroSimE) (pp. 1-8). IEEE.

7. Bailey, C., Stoyanov, S., Tilford, T. and Tourloukis, G., 2016, January. 3D-printing and electronic packaging. In 2016 Pan Pacific Microelectronics Symposium (Pan Pacific) (pp. 1-7). IEEE.

8. Teng, K. and Vest, R.W., 1987. Liquid ink jet printing with MOD inks for hybrid microcircuits. IEEE Transactions on Components, Hybrids, and Manufacturing Technology, 10(4), pp.545-549.

9. Dearden, A.L., Smith, P.J., Shin, D.Y., Reis, N., Derby, B. and O'Brien, P., 2005. A low curing temperature silver ink for use in ink-jet printing and subsequent production of conductive tracks. Macromolecular Rapid Communications, 26(4), pp.315-318.

10. Smith, P.J., Shin, D. Y., Stringer, J.E., Derby, B. and Reis, N., 2006. Direct ink-jet printing and low temperature conversion of conductive silver patterns. Journal of Materials Science, 41(13), pp.4153-4158.

11. Teng, K.F., Azadpour, M.A. and Yang, H.Y., 1988, May. Rapid prototyping of multichip packages using computer-controlled, ink jet direct-write. In 38th Electronics Components Conference 1988., Proceedings. (pp. 326-329). IEEE.

12. Dziedzic, A., Nijs, J. and Szlufcik, J., 1993. Thick-film Fine-line Fabrication Techniques-Application to Front Metallisation of Solar Cells. Microelectronics International, 10(1), pp.18-26.

13. Sirringhaus, H., Kawase, T., Friend, R.H., Shimoda, T., Inbasekaran, M., Wu, W. and Woo, E.P., 2000. High-resolution inkjet printing of allpolymer transistor circuits. Science, 290(5499), pp.2123-2126.

14. Burns, S.E., Cain, P., Mills, J., Wang, J. and Sirringhaus, H., 2003. Inkjet printing of polymer thin-film transistor circuits. MRS bulletin, 28(11), pp.829-834.

15. Van Osch, T.H., Perelaer, J., de Laat, A.W. and Schubert, U.S., 2008. Inkjet printing of narrow conductive tracks on untreated polymeric substrates. Advanced Materials, 20(2), pp.343-345.

16. Eom, S.H., Senthilarasu, S., Uthirakumar, P., Yoon, S.C., Lim, J., Lee, C., Lim, H.S., Lee, J. and Lee, S.H., 2009. Polymer solar cells based on inkjet-printed PEDOT: PSS layer. Organic Electronics, 10(3), pp.536542.

17. Eom, S.H., Park, H., Mujawar, S.H., Yoon, S.C., Kim, S.S., Na, S.I., Kang, S.J., Khim, D., Kim, D.Y. and Lee, S.H., 2010. High efficiency polymer solar cells via sequential inkjet-printing of PEDOT: PSS and P3HT: PCBM inks with additives. Organic Electronics, 11(9), pp.15161522.

18. Szczech, J., Megaridis, C., Zhang, J. and Gamota, D., 2004. Ink jet processing of metallic nanoparticle suspensions for electronic circuitry fabrication. Microscale Thermophysical Engineering, 8(4), pp.327-339.

19. Bieri, N.R., Chung, J., Poulikakos, D. and Grigoropoulos, C.P., 2004 Manufacturing of nanoscale thickness gold lines by laser curing of a discretely deposited nanoparticle suspension. Superlattices and Microstructures, 35(3-6), pp.437-444.

20. Chung, J., Ko, S., Bieri, N.R., Grigoropoulos, C.P. and Poulikakos, D., 2004. Conductor microstructures by laser curing of printed gold nanoparticle ink. Applied Physics Letters, 84(5), pp.801-803.

21. Ko, S.H., Chung, J., Choi, Y., Grigoropoulos, C.P., Bieri, N.R., Choi, T.Y., Dockendorf, C. and Poulikakos, D., 2005, April. Laser based hybrid inkjet printing of nanoink for flexible electronics. In Photon Processing in Microelectronics and Photonics IV (Vol. 5713, pp. 97-104). International Society for Optics and Photonics.

22. Ko, S.H., Chung, J., Pan, H., Grigoropoulos, C.P. and Poulikakos, D., 2006, March. Fabrication of multilayer passive electric components using inkjet printing and low temperature laser processing on polymer. In Photon Processing in Microelectronics and Photonics V (Vol. 6106, p. 61060E). International Society for Optics and Photonics.

23. Perelaer, J., De Gans, B.J. and Schubert, U.S., 2006. Ink-jet printing and microwave sintering of conductive silver tracks. Advanced Materials, 18(16), pp.2101-2104

24. Adamietz, R., Desmulliez, M.P., Pavuluri, S.K., Tilford, T., Bailey, C., Schreier-Alt, T. and Warmuth, J., 2018. Reliability testing and stress measurement of QFN packages encapsulated by an open-ended microwave curing system. IEEE Transactions on Components, Packaging and Manufacturing Technology, 9(1), pp.173-180.

25. Pavuluri, S.K., Ferenets, M., Goussetis, G., Desmulliez, M.P., Tilford, T., Adamietz, R., Mueller, G., Eicher, F. and Bailey, C., 2012. Encapsulation of microelectronic components using open-ended microwave oven. IEEE Transactions on Components, Packaging and Manufacturing Technology, 2(5), pp.799-806.

26. Tilford, T., Sinclair, K.I., Bailey, C., Desmulliez, M.P., Goussettis, G., Parrott, A.K. and Sangster, A.J., 2007. Multiphysics simulation of microwave curing in micro-electronics packaging applications. Soldering \& Surface Mount Technology, 19(3), pp.26-33.

27. Sinclair, K.I., Goussetis, G., Desmulliez, M.P., Sangster, A.J., Tilford, T., Bailey, C. and Parrott, A.K., 2008. Optimization of an open-ended microwave oven for microelectronics packaging. IEEE transactions on microwave theory and techniques, 56(11), pp.2635-2641.

28. Torrisi, F., Hasan, T., Wu, W., Sun, Z., Lombardo, A., Kulmala, T.S., Hsieh, G.W., Jung, S., Bonaccorso, F., Paul, P.J. and Chu, D., 2012. Inkjet-printed graphene electronics. ACS nano, 6(4), pp.2992-3006.

29. Li, J., Ye, F., Vaziri, S., Muhammed, M., Lemme, M.C. and Östling, M., 2013. Efficient inkjet printing of graphene. Advanced materials, 25(29), pp.3985-3992.

30. Tortorich, R. and Choi, J.W., 2013. Inkjet printing of carbon nanotubes. Nanomaterials, 3(3), pp.453-468.

31. Chen, K., Gao, W., Emaminejad, S., Kiriya, D., Ota, H., Nyein, H.Y.Y., Takei, K. and Javey, A., 2016. Printed carbon nanotube electronics and sensor systems. Advanced Materials, 28(22), pp.4397-4414.

32. Zhang, Y., Liu, C. and Whalley, D.C., Direct-write techniques for maskless production of microelectronics: a review of current state-of-theart technologies, Proc International Conference on Electronic Packaging Technology \& High Density Packaging, (ICEPT-HDP), 2009, Beijing, China, 10-13 Aug., pp. 497-503

33. Cummins, G, and Desmulliez, M.P.Y,. Inkjet printing of conductive materials: a review, Circuit World, 38/4 (2012) 193-213

34. Wünscher, S., Rasp, T., Grouchko, M., Kamyshny, A., Paulus, R.M., Perelaer, J., Kraft, T., Magdassi, S. and Schubert, U.S., 2014. Simulation and prediction of the thermal sintering behavior for a silver nanoparticle ink based on experimental input. Journal of Materials Chemistry C, 2(31), pp.6342-6352.

35. Miettinen, J., Pekkanen, V., Kaija, K., Mansikkamäki, P., Mäntysalo, J., Mäntysalo, M., Niittynen, J., Pekkanen, J., Saviauk, T. and Rönkkä, R., 2008. Inkjet printed system-in-package design and manufacturing. Microelectronics Journal, 39(12), pp.1740-1750.

36. Kaija, K., Pekkanen, V., Mäntysalo, M., Koskinen, S., Niittynen, J., Halonen, E. and Mansikkamäki, P., 2010. Inkjetting dielectric layer for electronic applications. Microelectronic Engineering, 87(10), pp.19841991.

37. Pekkanen, V., Mäntysalo, M., Kaija, K., Mansikkamäki, P., Kunnari, E., Laine, K., Niittynen, J., Koskinen, S., Halonen, E. and Caglar, U., 2010. Utilizing inkjet printing to fabricate electrical interconnections in a system-in-package. Microelectronic Engineering, 87(11), pp.2382-2390.

38. Pynttari, V., Halonen, E., Sillanpaa, H., Mantysalo, M. and Makinen, R., 2012. RF design for inkjet technology: Antenna geometries and layer thickness optimization. IEEE Antennas and Wireless propagation letters, 11, pp.188-191.

39. Xie, L., Yang, G., Mantysalo, M., Xu, L.L., Jonsson, F. and Zheng, L.R., 2012. Heterogeneous integration of bio-sensing system-on-chip and printed electronics. IEEE Journal on Emerging and Selected Topics in Circuits and Systems, 2(4), pp.672-682

40. Vuorinen, T., Vehkaoja, A., Jeyhani, V., Noponen, K., Onubeze, A., Kankkunen, T., Puuronen, A.K., Nurmentaus, S., Preejith, S.P., Joseph, J. and Seppänen, T., 2016, September. Printed, skin-mounted hybrid system for ECG measurements. In 2016 6th Electronic System-Integration Technology Conference (ESTC) (pp. 1-6). IEEE.

41. Kawahara, Y., Hodges, S., Cook, B.S., Zhang, C. and Abowd, G.D., 2013, September. Instant inkjet circuits: lab-based inkjet printing to support rapid prototyping of UbiComp devices. In Proceedings of the 2013 ACM international joint conference on Pervasive and ubiquitous computing (pp. 363-372). ACM

42. T. Falat, J. Felba, A. Moscicki and J. Borecki, "Nano-silver inkjet printed interconnections through the microvias for flexible electronics," 2011 11th IEEE International Conference on Nanotechnology, Portland, OR, 2011, pp. 473-477.

43. Felba, J., 2011, May. Inkjet printed electrically conductive structures for microelectronics. In Proceedings of the 2011 34th International Spring Seminar on Electronics Technology (ISSE) (pp. 6-11). IEEE. 
44. L. Faller and H. Zangl, "Robust design of a 3D- and inkjet-printed capacitive force/pressure sensor," 2016 17th International Conference on Thermal, Mechanical and Multi-Physics Simulation and Experiments in Microelectronics and Microsystems (EuroSimE), Montpellier, 2016, pp. $1-7$.

45. Faller, L.M. and Zangl, H., 2017, May. Robust design of an inkjet-printed capacitive sensor for position tracking of a MOEMS-mirror in a Michelson interferometer setup. In Smart Sensors, Actuators, and MEMS VIII (Vol. 10246, p. 1024611). International Society for Optics and Photonics.

46. Faller, L.M., Granig, W., Krivec, M., Abram, A. and Zangl, H., 2018. Rapid prototyping of force/pressure sensors using 3D-and inkjet-printing. Journal of Micromechanics and Microengineering, 28(10), p.104002.

47. Khan, Y., Ostfeld, A.E., Lochner, C.M., Pierre, A. and Arias, A.C., 2016. Monitoring of vital signs with flexible and wearable medical devices. Advanced Materials, 28(22), pp.4373-4395

48. Khan, Y., Garg, M., Gui, Q., Schadt, M., Gaikwad, A., Han, D., Yamamoto, N.A., Hart, P., Welte, R., Wilson, W. and Czarnecki, S., 2016. Flexible hybrid electronics: Direct interfacing of soft and hard electronics for wearable health monitoring. Advanced Functional Materials, 26(47), pp.8764-8775

49. Leber, D.E., Chaney, R.L., Hackler, D.R., Meek, B.N., Leija, S.D., DeGregorio, K.J., Wald, S.F. and Wilson, D.G., 2017, April. Advances in flexible hybrid electronics reliability. In 2017 IEEE Workshop on Microelectronics and Electron Devices (WMED) (pp. 1-4). IEEE

50. https://www.nextflex.us [Accessed: 19 November 2020]

51. hen, R., Chow, J.H., Taylor, C., Meth, J. and Sitaraman, S.K., 2020. Mechanical and Electrical Behavior of Printed Silver Conductor in Adaptive Curvature Flexure Test. IEEE Transactions on Components, Packaging and Manufacturing Technology, 10(5), pp.806-816.

52. Lall, P., Narangaparambil, J., Leever, B. and Miller, S., 2019, May. Effect of Sintering Temperature on the Fatigue Life of Additively Printed Electronics During Cyclic Bending. In 2019 18th IEEE Intersociety Conference on Thermal and Thermomechanical Phenomena in Electronic Systems (ITherm) (pp. 189-197). IEEE.

53. Roshanghias, A., Krivec, M. and Baumgart, M., 2017. Sintering strategies for inkjet-printed metallic traces in 3D printed electronics. Flexible and Printed Electronics, 2(4), p.045002.

54. http://www.nano-di.com [Accessed: 3 January 2020]

55. O'Reilly, M. and Leal, J., 2010. Jetting your way to fine-pitch 3D interconnects. Chip Scale Review, 14(5), pp.18-21.

56. http://www.nextfactory-project.eu [Accessed: 3 January 2020]

57. www.jedec.org [Accessed: 3 January 2020]

58. Tilford, T., Stoyanov, S., Braun, J., Janhsen, J. C., Burgard, M., Birch, R. and Bailey, C., Design, manufacture and test for reliable 3D printed electronics packaging. Microelectronics Reliability, 2018, Vol. 85 pp. 109-117

59. Bailey, C., Stoyanov, S., Tilford, T. and Tourloukis, G., 2017, April. Multi-physics models and condition-based monitoring for 3d-printing of electronic packages. In 2017 18th International Conference on Thermal, Mechanical and Multi-Physics Simulation and Experiments in Microelectronics and Microsystems (EuroSimE) (pp. 1-8). IEEE.

60. Properties of Bulk Sintered Silver as a Function of Porosity, A. A Wereszczak, D. J. Vuono, H Wang, M. K. Ferber and Z. Liang, ORNL/TM-2012/130, June 2012

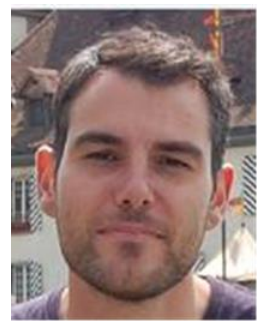

Tim Tilford obtained a Bachelor's degree in Aeronautical Engineering from Queen Mary College, University of London, United Kingdom, in 1998 and subsequently a Master's Degree in Computational Fluid Dynamics from the University of Greenwich, United Kingdom, in 1999. He obtained a $\mathrm{PhD}$ in Computational Mechanics from the University of Greenwich in 2013. He has been a Research Fellow and subsequently a Senior Lecturer at the University of Greenwich since 2002. His primary research interests are in numerical analysis of multiphysics/multi-scale problems and high-performance parallel computing. Dr. Tilford is a Fellow of the Institute of Mathematics and its Applications, a Chartered Mathematician and a Chartered Scientist

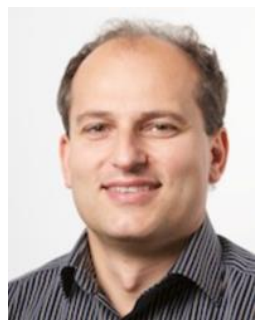

Stoyan Stoyanov received the Ph.D. degree in optimization modeling for microelectronics packaging and product design from the University of Greenwich, London, U.K., in 2004. He is a Senior Research Fellow within the Computational Mechanics and Reliability Group, School of Computing and Mathematical Sciences, University of Greenwich. His research interests are in the field of simulation based methodologies that exploit the integration of optimization and statistical techniques with methods for multiphysics analysis, with applications in reliable, robust, high performance and low cost design of electronic products and components and identification of optimal conditions for the related manufacturing processes.

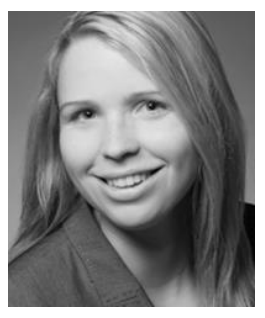

Jessica Braun studied Bioprocess engineering in Dresden and works as Research Engineer and Project Manager in the department Additive Manufacturing at Fraunhofer IPA since 2013. Her working fields include the design and development of printing processes for functional printing in 2D and 3D applications. This especially relates to piezo-inkjet technology including ink conditioning, optimization, tests and validation.

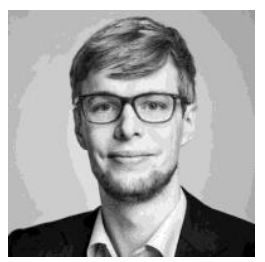

Jan Christoph Janhsen studied mechanical engineering in Aachen. After his Master of Engineering he started working at Fraunhofer IPA 2015. His work topics include the design of control software and automation for 3D printing machines and test rigs with a focus on photopolymerbased AM processes and hybrid process combinations for AMbased manufacturing technologies. Since the beginning of 2020 he is group manager for the group 'Photopolymer-based AM Processes' in the department Additive Manufacturing at Fraunhofer IPA.

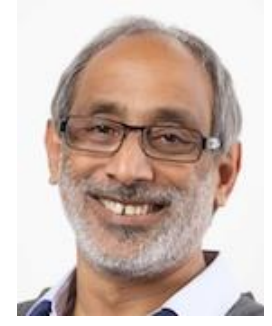

Mayur K Patel is a Professor of Computational Science and Engineering and the Faculty Director for Partnerships and Internationalization, which currently spans four schools and over 20 countries, with the University of Greenwich, London, U.K, He has published over 150 publications in the field of Computational Fluid Dynamics, its development and application in a wide range of industries, over a period of 40 years and has worked with a number of organisations in the UK and overseas. Prof. Patel is a Fellow of the Institute of Mathematics and its Applications a Chartered Mathematician and a Chartered Scientist. 
Chris Bailey (M'92-SM'06) received the $\mathrm{Ph} . \mathrm{D}$. degree in computational modeling and the M.B.A. degree in technology management in 1988 and 1996, respectively. $\mathrm{He}$ is a Professor of computational mechanics and reliability with the University of Greenwich, London, U.K, and the Director of the Computational Mechanics and Reliability Group. He has published over 200 papers on design and simulation of micro/nanotechnology-based processes and products and has worked on many U.K. and European Union projects. His current research interests include multiphysics/scale modeling of micro-nanosystems, predictive reliability, and prognostics. Prof. Bailey is President of the IEEE Electronics Packaging Society and a Committee Member of the International Microelectronics and Packaging Society, U.K. 